dem er als Experte wirkt. 1983 verlieh ihm der Deutsche Jagdschutz-Verband den Kulturpreis, 1977 erhielt er das Verdienstkreuz des Niedersächsischen Verdienstordens.

Dem Kreis der Jagdwissenschaftler ist der Jubilar seit eh und je verbunden. Mit seiner charmanten und liebenswerten Art möge er noch recht lange unseren Kreis bereichern.

ERHARD UECKERMANN

\title{
Prof. Dr. Rieck wurde 85 Jahre
}

In seinem Heim in Hann. Münden beging WaLTER Rreck, am 1. Februar 1905 in Prenzlau in der Uckermark geboren, seinen 85. Geburtstag. Sein Wirken als herausragender Jagdwissenschaftler spiegelt den Wechsel in der jüngeren deutschen Geschichte besonders deutlich wider. Von 1937 bis 1945 leitete er das Institut für Jagdkunde des Reichsbundes Deutsche Jägerschaft, wohl für das gesamte damalige Reichsgebiet zuständig, aber doch mit einem Arbeitsschwerpunkt im Berliner Raum und der Mark Brandenburg. Als solcher initiierte er die Herausgabe der Zeitschrift für Jagdkunde (von 1939 bis 1944 erschienen), die ab 1955 in der Zeitschrift für Jagdwissenschaft fortgeführt und von ihm als Schriftleiter bis 1972 betreut wurde.

Mit seinem Eintritt in das Institut für Jagdkunde der Universität Göttingen im Jahre 1950 begannen viele Jahre des Wirkens für die Jäger in der Bundesrepublik, die u. a. 1962 zur Ernennung zum Professor und zum Institutsleiter von 1968 bis 1972 führten. Seine Arbeiten waren stets praxisbezogen und sollen an dieser Stelle nur durch die Themenbereiche Hasenforschung, Wildmarkenforschung, Altersschätzung, Wildkunde Rehwild und Wildkrankheiten belegt werden. In Heft 1/1985 der Zeitschrift für Jagdwissenschaft wurde sein Wirken anläßlich der Vollendung seines 80 . Lebensjahres auf den ersten beiden Seiten dargestellt und gewürdigt.

Ein herzlicher Dank geht von der Jägerschaft und dem Kollegenkreis zu diesem Lebensabschnitt an Prof. Dr. Rieck, der umfassend und grundlegend das Waidwerk in unserem Vaterlande förderte, verbunden mit allen guten $W$ ünschen, insbesondere für seine Gesundheit.

ERHARD UECKERMANN

\section{Arbeitstagung Fütterung und Äsungsverbesserung für Reh- und Rotwild in Aulendorf}

Dr. M. Prgel, Leiter der Wildforschungsstelle des Landes Baden-Württemberg in Aulendorf, hatte für die Zeit vom 13. bis 15. Dezember 1989 in sein Institut nach Aulendorf zu einer Diskussion von Fragen zur Äsungsverbesserung mit dem Schwerpunkt der Fütterung eingeladen. Im Kreis von Fachwissenschaftlern aus Österreich und der Bundesrepublik Deutschland erörterten Vertreter des Landesjagdverbandes Baden-Württemberg (Präsidiumsmitglied Forstdirektor OESTERREICH und Bezirksjägermeister GüNTHER) und des Ministeriums für Ländlichen Raum, Landwirtschaft und Forsten (Ministerialrat WEIDEN$\mathrm{BACH}$, Landesforstverwaltung, und Regierungsdirektor SCHNIEPP, Jagdreferent) gesetzliche, ökologische, ernährungsphysiologische und technische Grundlagen der Wildfütterung, wobei ein wesentliches Ziel der Veranstaltung die Erarbeitung eines Merkblattes seitens der Wildforschungsstelle des Landes Baden-Württemberg war, das sowohl von der Landesforstverwaltung als auch der Landesjägerschaft getragen werden kann. Im Lande bestehende Auffassungsunterschiede sollten auf diese Weise objektiviert und angenähert werden. 